Somnologie $2011 \cdot 15: 133-134$

DOI 10.1007/s11818-011-0529-6

Online publiziert: 25. August 2011

(c) Springer-Verlag 2011

\author{
G. Mayer \\ Hephata-Klinik, Schwalmstadt
}

\title{
Schlafmedizinischer Versorgungsbedarf in Deutschland
}

die Spitze der europäischen Schlafmedizin gesetzt. Aus den Initiativen deutscher Schlafforscher haben sich Netzwerke für das Restless-Legs-Syndrom (EURLSSG), die Insomnien (European Insomnia Network), die Narkolepsie und ihre Grenzgebiete (European Narcolepsy Network) sowie die REM-Schlafverhaltensstörung (International RBD Study Group) gebildet.

Die rasche Entwicklung hatte aber auch einige Nachteile zur Folge: Im Rahmen der rasch wachsenden Untersuchungszahlen haben sich $u$. a. die Erlöse für schlafmedizinische Prozeduren stark verringert, und es ist bisher noch nicht gelungen, die Erlössituation zu verbessern. Ambulante Untersuchungen werden von der Gesundheitspolitik gefordert und stationäre, etablierte Strukturen hierdurch gefährdet, auch wenn sie nachweislich nicht teurer sind. Die Schlafforschung hat die Anerkennung als eigenständige wissenschaftliche Disziplin mit eigenem Lehrstuhl noch nicht erreicht, wenngleich inzwischen eine Stiftungsprofessur eingerichtet wurde. Die klinische Schlafmedizin ist nur in wenigen interdisziplinären Arbeitsbereichen oder Abteilungen aufgestellt, nachhaltige Strukturen gibt es jedoch noch nicht. Die Anzahl universitärer Curricula ist verschwindend gering und verfügt über zu wenige Stundenzahlen. Junge Wissenschaftler mit schlafmedizinischen Themen können noch keine wissenschaftlichen Karrieren an deutschen Universitäten anstreben, da es nur sehr wenige Abteilungen mit einem Schwerpunkt Schlafmedizin gibt. Die Grundlagenforschung kann sich nur dann neben der klinischen Routine durchsetzen, wenn sie von einzelnen Lehrstuhlinhabern explizit gefördert wird. In der Folge gehen junge Wissenschaftler, die im Ausland neue Methoden und schlafmedizinisches Wissen erworben haben, der deutschen Schlafmedizin und Schlafforschung verloren.

Um dieser negativen Entwicklung entgegen zu wirken, bedarf es neuer Konzepte und Initiativen. Definitionen und Strukturen, wie sie von der DGSM gefordert werden, sollen nicht hemmen, sondern Entwicklungen fördern, Qualität sichern und für Transparenz nach innen und außen sorgen. Qualität funktioniert nicht ohne Spezialisten, und sie kostet zunächst einmal Geld. Umgekehrt kann die Etablierung der Qualität aber für Kosteneindämmung sorgen, indem Spezialisten und nicht allgemeine Bestimmungen (z. B. BUB-Richtlinien, Bewertung ärztlicher Untersuchungs- und Behandlungsmethoden) darüber entscheiden, was für den einzelnen Patienten wann erforderlich ist. Die Schlafmedizin muss in dieser Hinsicht Partner der Kostenträger werden. Das ist bisher aber nur äußerst selten geschehen.

\section{(? Qualität kann für Kosteneindämmung sorgen}

Schlafmedizin ist, wie uns die Zahlen für das erhöhte Risiko für metabolische- und Herz-Kreislauf-Erkrankungen sowie eine erhöhte Mortalität durch kurze Schlafzeiten zeigen [6], eine Fachdisziplin, die ihren Einsatz in der Krankheitsprävention offenkundig macht. 
Standen laut Statistischem Bundesamt stationär behandelte Schlafstörungen (ICD-10: G47/F51) mit 120.289 Fällen 2006 noch an 9. Stelle aller Erkrankungen, so lagen sie 2009 auf Platz 49 (o,4\%). Bei niedergelassenen Ärzten liegen sie seit 2002 unverändert auf Platz 27, in Nervenarztpraxen Platz 23. Klassischerweise liegen die schlafbezogenen Atmungsstörungen 2006 (Gesundheitsberichtserstattung des Bundes) mit 117.936 stationär behandelten Fällen an einsamer Spitze, gefolgt von 1472 Fällen mit Hypersomnie und 610 Fällen mit Insomnie. Gerade diese letzte Zahl zeigt ein erschreckendes Versorgungsdefizit an. Die Prävalenz der Insomnie liegt bei bis zu 25\% der Bevölkerung [5], und hinter einem Viertel dieser Fälle verbergen sich andere, z. T. schwerwiegende Erkrankungen, die nur mithilfe der Polysomnographie diagnostiziert werden können [7, 8]. Einen eindrucksvollen Beleg für die Bedeutung der Insomnien gibt der DAKGesundheitsreport 2010 [1], der darüber hinaus zeigt, dass hochgradige Schlafstörungen besonders häufig vor dem Hintergrund anderer Gesundheitsstörungen wie Herz-Kreislauf-, Schmerz-, psychiatrischen, Lungen-, neurologischen Erkrankungen und Adipositas (in dieser Häufigkeitsfolge) auftreten. Zusammenfassend kommt der DAK-Report zu der Schlussfolgerung, dass

- etwa 10\% der aktiv Erwerbstätigen unter behandlungsbedürftigen Schlafstörungen leiden,

- Schlafstörungen und damit einhergehende Gesundheitsfolgen von den Betroffenen unterschätzt werden und in der medizinischen Versorgung nicht sofort erkannt werden,

- qualitätssteigernde Aktivitäten im Bereich der schlafmedizinischen Diagnostik und Therapie verstärkt werden müssen.

Die Wissenschaftler können diese Erkenntnisse der Kassen bestätigen und sehen darin ein Zeichen und eine Möglichkeit, gemeinsam an diesen Zielen mitzuwirken. Aus der wissenschaftlichen Erkenntnis und den sich daraus ergebenden Konsequenzen folgt unmittelbar, wie die zukünftigen Schritte der Schlafmedizin aussehen müssen:
- Schaffung von schlafmedizinischen Professuren und Lehrstühlen an den Universitäten,

- Aufbau von Grundlagenforschung,

- Erweiterung der schlafmedizinischen Diagnostik und Therapie für das gesamte Spektrum der Schlafmedizin unter Einbeziehung und Honorierung von Beratung, Behandlung und Langzeitbetreuung klinisch diagnostizierbarer schlafmedizinischer Erkrankungen.

Als weitere Ziele ergeben sich:

- Verbesserung der schlafmedizinischen Ausbildung für Allgemeinund Fachärzte,

- Verbesserte Informationsstruktur bei den Krankenkassen, Patienten und Politikern,

- Prävention von Schlafstörungen mittels

- besserer Zusammenarbeit mit Betriebsärzten und Arbeitsmedizinern im Rahmen der betrieblichen Gesundheitsprävention,

- Aufklärung über Auswirkung von insuffizientem Schlaf und Schlafstörungen auf Leistungsfähigkeit, Unfallneigung, Folgeerkrankungen,

- früher Diagnostik und Therapie von Schlafstörungen, die den Schlaf erheblich beeinträchtigen,

- Prädiktorenentwicklung für Therapien.

Gestörter Schlaf ist letztendlich mehr als nur eine Befindlichkeitsstörung und wir werden weiter daran arbeiten müssen, um das Gesundheitswesen darauf aufmerksam zu machen!

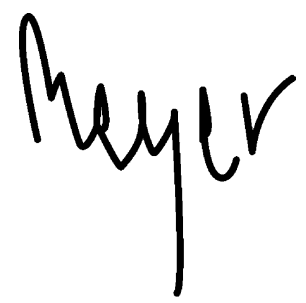

G. Mayer, DGSM-Vorstand

\section{Korrespondenzadresse}

Prof. Dr. G. Mayer

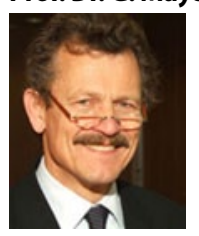

Hephata-Klinik

Schimmelpfengstr. 2,

34613 Schwalmstadt

dgsm.mayer@t-online.de

\section{Literatur}

1. DAK (2010) DAK-Gesundheitsreport 2010, IGES Institut GmbH, Berlin. http://wwwpresse.dak.de/ps.nsf/Show/ 03AF73C39B7227B0C12576BF004C8490/\$File/ DAK_Gesundheitsreport_2010_2402.pdf. Zugegriffen: 04.08.11

2. Fischer J, Mayer G, Peter HJ, Riemann D, Sitter H (2001) Leitlinie "S2" der Deutschen Gesellschaft für Schlafforschung und Schlafmedizin (DGSM): Nichterholsamer Schlaf. Somnologie5(Suppl 3):1-258

3. Gesundheitsberichtserstattung des Bundes (2011) Homepage. http://www.gbe-bund.de/gbe10/ pkg_isgbe5.prc_isgbe?p_uid=gastd. Zugegriffen: 04.08.11

4. Mayer G, Fietze I, Fischer J et al (2009) S3-Leitlinie "Nicht erholsamer Schlaf/Schlafstörungen". Somnologie13(Suppl 1):1-160

5. Ohayon, M (2002) Epidemiology of insomnia: what we know and what we still need to learn. Sleep Med Rev 6:97-111

6. Gallicchio L, Kalesan B(2009) Sleep duration and mortality: a systematic review and meta-analysis. J Sleep Res 18:148-158

7. Reite M, Buysse DJ, Reynolds CF, Mendelson WB (1995) The use of polysomnography in the evaluation of insomnia. Sleep 18:58-70

8. Taylor DJ, Mallory LJ, Lichstein KL et al (2007) Comorbidity of chronic insomnia with medical problems. Sleep 30(2):213-218 